\title{
Equilibrium Pricing in Incomplete Markets
}

\author{
Abdelhamid Bizid and Elyès Jouini*
}

\begin{abstract}
Given exogenously the price process of some assets, we constrain the price process of other assets, which are characterized by their final pay-offs. We deal with an incomplete market framework in a discrete time model and assume the existence of the equilibrium. In this setup, we derive restrictions on the state-price deflators and these restrictions do not depend on a particular choice of utility function. A stochastic volatility model is numerically investigated as an example. Our approach leads to an interval of admissible prices much better than the arbitrage pricing interval.
\end{abstract}

\section{Introduction}

Pricing of contingent claims takes its roots in the pioneering works of Black and Scholes (1973) and Harrison and Kreps (1979). Their results are based on the key idea that the prices of the existing assets induce a unique arbitrage-free price for any new redundant asset. When it is not redundant, the price must however lie in an arbitrage-free interval. A large part of the literature has dealt with the reduction of this interval in order to restrict the bid-ask spread and to obtain an unambiguous price. An important insight is due to Bensaid, Lesne, Pages and Scheinkman (1992) who introduced the super-replication cost in a transaction costs framework. They gave more accurate upper and lower bounds for the price of an asset than the ones obtained by the replicating strategies with simply requiring the price functional to be non-decreasing with respect to the pay-off. Unfortunately, even with those restrictions, the bid-ask spread remains, in general, too large as showed in Soner, Shreve and Cvitanić (1995). An alternative approach has been suggested by Föllmer and Schweizer (1991), where the agents are assumed to minimize the local quadratic risk. This approach leads to a unique price for a given derivative security but the assumption is very restrictive. In a

\footnotetext{
${ }^{0}$ Bizid, CDC-Marchés, CDC Group and Université Paris I - Sorbonne ; Jouini, Université Paris IX - Dauphine. INQUIRE-Europe financial support is gratefully acknowledged.
} 
transaction costs framework and in order to obtain bounds without a particular choice of utility function, Constantinides (1993) considers the class of power functions, with restrictions on the relative risk aversion coefficient. He obtains bounds on the option prices depending on the restrictions and valid for the considered class of utility functions.

In our paper, we propose a new approach by exploiting partial conditions issued from the equilibrium analysis. We assume that the price processes of some assets are given exogenously (e.g. follow from econometric estimations) and we want to constrain the price processes of other assets (typically, derivative assets) characterized by their value at maturity. We a priori deal with an incomplete market framework and we obtain bounds on the density of the pricing probabilities and thereafter, on the asset prices. Our initial assumptions are similar to the Consumption-based Capital Asset Pricing Model (CCAPM) of Rubinstein (1976) and Breeden and Litzenberger (1978). However, our results are valid for all the Von Neumann-Morgenstern utility functions, without any restriction on the riskaversion.

Let us go further in this comparison with the CCAPM. By assuming the market completeness, the CCAPM allows one to link State-Price Deflators (or Pricing Probabilities) with Global Consumption, through the existence of a representative agent. Furthermore, in order to derive explicit pricing formulae in discrete time, one should first make an explicit choice for the individuals' utility functions and then, estimate the aggregate consumption in the economy. In Breeden, Gibbons and Litzenberger (1989), the authors point out the main measurement problems for this aggregate consumption process : "1) the reporting (by the Department of Commerce) of expenditures, rather than consumption, 2) the reporting of an integral of consumption rate rather than the consumption rate at a point in time, 3) infrequent reporting of consumption data relative to stock returns, and 4) reporting aggregate consumption with sampling error since only a subset of the total population of consumption transactions is measured."

In order to avoid such an explicit choice of a utility function, we replace the covariance approach used in the CCAPM by an ordering approach valid for all concave VNM utility functions. This is the main concern of section III, in this paper. Moreover, the well-known equity premium puzzle documented by Mehra and Prescott (1985) shows the failure of the pure CCAPM approach. Zhou (1999) gives a good review of the papers, which try to solve this problem, and gives also a solution based on market incompleteness. This paper gives a procedure in an incomplete framework, in order to restrain the pricing bounds of derivative assets. 
In order to eliminate the aggregate consumption process from our analysis, we identify this process with the global dividend process through the market clearing conditions on the single consumption good market. Then, we assume that the dividends process and the market index process are similarly ordered. This is the main assumption of section $\mathrm{V}$, which deals with a practical application in an uncertain volatility model (as in Avellaneda, Levy and Paras (1995)). Note that, in Jouini and Napp (1999), it is shown that, for a large class of utility functions, there exists at least one equilibrium for which this assumption is satisfied. From a practical point of view, this assumption permits one to replace the consumption process by the total wealth process or the market index one (seen as a proxy of the total wealth). Such a substitution is compatible with the empirical conclusions of Mankiw and Shapiro (1986): "... it seems possible that the CCAPM holds for the minority of consumers that hold stock and that our stock market index is a better proxy for the consumption of this minority than is aggregate consumption". $>$ From a theoretical point of view, this assumption is also compatible with the conclusions of Huang and Litzenberger (1988, p.211) under the Markov condition. Finally, if we want to get rid of this assumption, it suffices to specify a global dividend process in order to obtain computational results. Note that, even in this last case, the observation of only two processes (the global dividend and the market index) is required, independently of the degree of incompleteness (or the number of sources of uncertainty), in order to obtain useful bounds on the equilibrium asset prices and restrictions on the equilibrium state price densities.

Our approach is related to the Payoff Distribution Pricing Model (PDPM) introduced by Dybvig $(1988 a, b)$, who considers the implications of the individual optimality conditions on the asset price, for agents with VNM preferences and derives conclusions in terms of ordering. This model has been extended in a friction market case (including incomplete markets) by Jouini and Kallal (1993), who show that this approach does not permit a reduction of the interval of the admissible prices. The main difference with the present work is that we use explicitly the market clearing conditions.

Perrakis and Ryan (1984) and Perrakis (1986) intuited restrictions on the family of probabilities used to price the derivative assets, without setting an economic model. In the first reference, the restriction is discussed for a single-period model only. In the second reference, it is taken as a primitive assumption, in a dynamic model, in order to obtain bounds on European and American options. In fact, the authors propose an ordering principle on the risk-neutral probabilities. Bizid, Jouini and Koehl (1999) established that the underlying economic assumption of 
Perrakis' papers is in fact that the market model is complete and that, at each node, the different transitions are equiprobable. In order to prove this result, they assume the existence of the equilibrium and use the characterization of the stateprice deflators given by the first-order conditions for the representative agent.

On the contrary, we give here some restrictive properties of the risk-neutral transition density for a general discrete-time model without any completeness assumption. These restrictions are derived from the equilibrium theory, and more precisely from the market clearing conditions. The true probability distribution is explicitly used and leads to a partial order principle which is less restrictive than the complete markets one. Even if the approach seems similar to Bizid, Jouini and Koehl (1999), a careful reader can notice that the framework and the results are completely different from those of Perrakis and Ryan (1984) or Bizid, Jouini and Koehl (1999). We recall that, in Perrakis and Ryan (1984), the market is complete even if we don't observe the completion (i.e. the assets which complete the market). Therefore, applying Perrakis and Ryan's (1984) computational method to an incomplete framework amounts to consider the equilibrium prices for all the admissible completions of the market. However, and contrary to the intuition given by the optimal investment-consumption problem (see e.g. Karatzas and Shreve (1998)), the pricing interval which is consistent with an equilibrium in an incomplete framework is wider than the one which is consistent with at least one completion. Therefore, from the equilibrium point of view, it appears that the asset pricing problem in an incomplete market is not equivalent to the same problem in a complete market with incomplete data. The specific approach developed in this paper for the incomplete market setting seems then to be worthy.

A simple example which shows the specificity of this approach is given in Section II. Then, the discrete-time dynamic model and the equilibrium restrictions are stated in Section III. There are $m+1$ assets: one equity claim with a total supply normalized to one and $m$ purely financial assets, each one with a total supply equal to zero. We can generalize this setting to a "multi assets" market where the claim is in fact the market index and where our methodology is applied to any asset (in zero or non-zero total supply) defined by its terminal payoff. Note also that the derivative assets are not specified (they can depend on some "projection" of the claim on some sources of uncertainty or even be uncorrelated with it). Furthermore, the derivative assets are not supposed to complete the market, as the degree of incompleteness is unknown. Agents have general Von Neumann-Morgenstern utility functions. We recall then the classical expression of the state-price deflators obtained by the individual first-order optimal conditions. 
We assume that the price process of the equity claim is exogenously known and we derive equilibrium restrictions on the state-price deflators. In Section IV, we take the price processes of the equity claim and of some purely financial assets as exogenous. As a special case, we consider the existence of a risk-free asset. We state our main result in terms of risk-neutral probabilities and show that we can narrow the admissible pricing interval if the information set is enlarged to other assets. In Section V, we investigate numerically a model with uncertain volatility and give various examples. The reduction of the bid-ask spread with respect to the arbitrage pricing interval (i.e. the interval, compatible with the no-arbitrage condition) justifies the interest of our approach.

This example has been previously studied in Bizid, Jouini and Koehl (1999) ; we give the interval of admissible prices (called "Perrakis' pricing interval" because the computing method is close to Perrakis' one) obtained by their approach (which considers the prices consistent with at least one completion of the market), together with our equilibrium pricing and the arbitrage pricing intervals. The equilibrium pricing interval appears significantly more precise than the arbitrage pricing one. Of course, Perrakis' pricing interval is always included in our equilibrium pricing interval, as it supposes also completeness of the market. The precision of Perrakis' pricing interval is linked to the ratio of the discounted expected value of the equity claim at maturity under the true probability, divided by its initial value. Owing to the risk-aversion of the agents, this ratio is always greater than one. Moreover, the nearer to one the ratio is, the finer the precision of this interval is. Note that Aït-Sahalia and Lo (1998) proposed this ratio as a measure of the market risk aversion. In an incomplete market, we find numerically that the nearer to one the ratio is, the wider the relative precision of our equilibrium pricing interval with respect to Perrakis' pricing interval is. On the contrary, it seems empirically that with a high market risk premium, our bounds are equal to Perrakis' ones. Therefore, our result could be interpreted as a justification of Perrakis' procedure for very risky market models.

\section{A Simple Example}

In order to explain our method of pricing, and before introducing our model, let us consider the simplest example of incomplete markets with three assets: a quadrinomial one-period model where the sample space and the probability are

$$
\Omega=\left\{\omega_{1}, \omega_{2}, \omega_{3}, \omega_{4}\right\}
$$




$$
P=(1 / 4,1 / 4,1 / 4,1 / 4)
$$

There are two assets in the market whose price processes are known : a primitive asset whose prices are

$$
\begin{aligned}
& p(0)=25 \text { at date } 0 \\
& p\left(\omega_{1}\right)=20, p\left(\omega_{2}\right)=30, p\left(\omega_{3}\right)=40 \text { and } p\left(\omega_{4}\right)=50 \text { at date } 1
\end{aligned}
$$

and a risk-free asset defined by an interest rate $r=\frac{1}{19}$.

It is straightforward that the feasible range of the densities of the martingale measures with respect to $P$ is such that :

$$
\begin{aligned}
& q\left(\omega_{1}\right)=\frac{7}{19}+q\left(\omega_{3}\right)+2 q\left(\omega_{4}\right) \\
& q\left(\omega_{2}\right)=\frac{12}{19}-2 q\left(\omega_{3}\right)-3 q\left(\omega_{4}\right)
\end{aligned}
$$

where $q\left(\omega_{i}\right)$ are positive for every $i$. The feasible risk-neutral probabilities are then in an area delimited by three points (in the simplex unity) :

$$
\begin{aligned}
& \left(\frac{7}{19}, \frac{12}{19}, 0,0\right) \\
& \left(\frac{15}{19}, 0,0, \frac{4}{19}\right) \\
& \left(\frac{13}{19}, 0, \frac{6}{19}, 0\right)
\end{aligned}
$$

Let us consider a completion of this market with non-redundant derivative assets in zero net supply. There exists a representative agent in this economy with a utility function $u$. Moreover, if there is one unit of primitive asset and no other endowment in the economy, the total wealth $W$ (of the representative agent) is equal to $p$.

At the equilibrium, the maximization program of this agent leads to the following first-order conditions:

$$
\begin{aligned}
& q\left(\omega_{1}\right)=\frac{u^{\prime}(20)}{u^{\prime}(25)} ; q\left(\omega_{2}\right)=\frac{u^{\prime}(30)}{u^{\prime}(25)} \\
& q\left(\omega_{3}\right)=\frac{u^{\prime}(40)}{u^{\prime}(25)} ; q\left(\omega_{4}\right)=\frac{u^{\prime}(50)}{u^{\prime}(25)} .
\end{aligned}
$$

Since $u$ is strictly concave, we obtain the following relationship, already induced by Perrakis

$$
q\left(\omega_{1}\right)>q\left(\omega_{2}\right)>q\left(\omega_{3}\right)>q\left(\omega_{4}\right)
$$


After imposing these restrictions, we get the feasible range of the risk-neutral probability delimited by the four following points :

$$
\begin{array}{ll}
Q_{1} & \left(\frac{11}{19}, \frac{4}{19}, \frac{4}{19}, 0\right) \\
Q_{2} & \left(\frac{26}{57}, \frac{26}{57}, \frac{5}{57}, 0\right) \\
Q_{3} & \left(\frac{12}{19}, \frac{2}{19}, \frac{2}{19}, \frac{2}{19}\right) \\
Q_{4} & \left(\frac{71}{152}, \frac{71}{152}, \frac{5}{152}, \frac{5}{152}\right)
\end{array}
$$

which is smaller than the range in the unrestricted case. Furthermore, note that these smaller bounds have been obtained under an existence assumption of the derivative assets but without specifying their price process. Since we deal with a linear pricing rule, the upper (resp. lower) bound on the equilibrium price of a given derivative asset defined by its payoff $x=\left(x_{1}, x_{2}, x_{3}, x_{4}\right)$ is reached at one of these four extremal points and we have then the following bounds :

$$
\left[\inf _{Q_{i}} E^{Q_{i}}(x) ; \sup _{Q_{i}} E^{Q_{i}}(x)\right]
$$

Consequently, this approach permits one to obtain bounds on the price of the derivative asset, which are better than the usual arbitrage-free bounds.

If we consider now directly our market and we do not assume anymore that this market is completed by some assets, we cannot apply the representative agent technique. However, we can find necessary conditions, at the equilibrium, on the risk-neutral probabilities. Using the maximization program of the $n^{\text {th }}$ agent, the density of his pricing probability with respect to $P$ must satisfy :

$$
\begin{aligned}
& q^{n}\left(\omega_{1}\right)=\frac{u_{n}^{\prime}\left(C^{n}\left(\omega_{1}\right)\right)}{u_{n}^{\prime}\left(C^{n}(0)\right)} ; q^{n}\left(\omega_{2}\right)=\frac{u_{n}^{\prime}\left(C^{n}\left(\omega_{2}\right)\right)}{u_{n}^{\prime}\left(C^{n}(0)\right)} \\
& q^{n}\left(\omega_{3}\right)=\frac{u_{n}^{\prime}\left(C^{n}\left(\omega_{3}\right)\right)}{u_{n}^{\prime}\left(C^{n}(0)\right)} ; q^{n}\left(\omega_{4}\right)=\frac{u_{n}^{\prime}\left(C^{n}\left(\omega_{4}\right)\right)}{u_{n}^{\prime}\left(C^{n}(0)\right)}
\end{aligned}
$$

where $C^{n}(\omega)$ denotes the optimal consumption of agent $n$ at the state of the world $\omega$. As, at date 1 , we have

$$
p\left(\omega_{i}\right)=\sum_{n} C^{n}\left(\omega_{i}\right)
$$


Since $p\left(\omega_{1}\right)<p\left(\omega_{2}\right)$, there exists at least one agent $n_{0}$ such that :

$$
C^{n_{0}}\left(\omega_{1}\right)<C^{n_{0}}\left(\omega_{2}\right)
$$

Since $u_{n_{0}}$ is strictly concave, we obtain that, for agent $n_{0}$

$$
q^{n_{0}}\left(\omega_{1}\right)>q^{n_{0}}\left(\omega_{2}\right)
$$

This restriction and the martingale measure condition leads to the fact that the feasible range of pricing probabilities must be in the area delimited by the four following points :

$$
\begin{array}{ll}
Q_{1}^{1,2} & \left(\frac{13}{19}, 0, \frac{6}{19}, 0\right) \\
Q_{2}^{1,2} & \left(\frac{26}{57}, \frac{26}{57}, \frac{5}{57}, 0\right) \\
Q_{3}^{1,2} & \left(\frac{15}{19}, 0,0, \frac{4}{19}\right) \\
Q_{4}^{1,2} & \left(\frac{9}{19}, \frac{9}{19}, 0, \frac{1}{19}\right)
\end{array}
$$

And the equilibrium price of a given derivative must belong to

$$
\left[\inf _{Q_{i}^{1,2}} E^{Q_{i}^{1,2}}(x) ; \sup _{Q_{i}^{1,2}} E^{Q_{i}^{1,2}}(x)\right]
$$

Using the same methodology for each pair $(i, j)$ such that $i<j$, we get that there exist five agents $n_{1}, n_{2}, n_{3}, n_{4}$ and $n_{5}$ such that :

$$
\begin{aligned}
& C^{n_{1}}\left(\omega_{1}\right)<C^{n_{1}}\left(\omega_{3}\right) \\
& C^{n_{2}}\left(\omega_{2}\right)<C^{n_{2}}\left(\omega_{3}\right) \\
& C^{n_{3}}\left(\omega_{1}\right)<C^{n_{3}}\left(\omega_{4}\right) \\
& C^{n_{4}}\left(\omega_{2}\right)<C^{n_{4}}\left(\omega_{4}\right) \\
& C^{n_{5}}\left(\omega_{3}\right)<C^{n_{5}}\left(\omega_{4}\right)
\end{aligned}
$$

leading to five other pricing intervals. Therefore the admissible interval of prices is necessarily the intersection of the six pricing intervals. The following graph represents the no-arbitrage probability set (the big triangle) and the different more restrictive sets (associated with each partial order). The intersection of these sets is the probability set for a complete market framework (Perrakis' assumption), as there is in fact a global order on the pricing probabilities. 


\section{FIGURE 1}

Intervals of the pricing probabilities

This Figure represents the six different intervals of pricing probabilities. Note that, since we deal with a quadrinomial framework, these intervals are characterized by the pair $\left(q\left(\omega_{3}\right), q\left(\omega_{4}\right)\right)$. The biggest triangle represents the no-arbitrage interval and the "black" interval (the intersection of the six intervals) is associated with Perrakis' assumption.

As an example, we price now a Call option with a strike equal to 35.

The classical no-arbitrage argument gives the following pricing bounds $[0 ; 3.16]$ at $t=0$. Perrakis' bounds are the following [0.44;2.11].

The incomplete market framework gives the following bounds $[0.44 ; 2.37]$ at date $t=0$. Therefore, even in an incomplete framework, we can obtain restrictions on option prices which are distinct from - but quite close to, the complete market framework. From a mathematical point of view, if we denote by $T_{i}, i=1, \ldots, 6$ the six different sets, Perrakis' bounds are given by :

$$
\left[\inf _{\cap T_{i}} E(x) ; \sup _{\cap T_{i}} E(x)\right]
$$

and our bounds by :

$$
\cap\left[\inf _{T_{i}} E(x) ; \sup _{T_{i}} E(x)\right]
$$

Even if this definition looks like the previous one, these sets are not equal unless the market is assumed to be complete. In such a situation, there is one agent which satisfies all the consumption constraints (in fact all the agents do). In other words, it is possible to take $n_{0}=n_{1}=\ldots=n_{5}$.

In the following, we consider a multi-period framework and explain how to use this recursive procedure for pricing derivatives in an incomplete discrete-time market.

\section{Framework and Equilibrium Restrictions}

In the following, we suppose that there is one nonstorable consumption good. For the sake of simplicity, we assume that this good is produced by only one firm. At each date $t$, the supply $d_{t}$ of this good is the production of the firm. This production is distributed as dividends to shareholders who own the firm. There is 
one equity claim which is tradable at date $t$ with post-dividend price $p_{t}$ in terms of consumption good, and perfectly divisible. After date $T$, the firm becomes obsolete and is valued at zero. A quantity $\theta$ of this claim insures to the owner a quantity $\theta d_{t}$ of the perishable good at date $t$. Throughout the paper, the total supply of the equity claim is normalized to one. Our results are not modified if we assume the existence of more than one productive asset. In that case, it would suffice to replace the process $\left(d_{t}\right)$ by the total dividend process and to assume, in the numerical examples (Section V), that this total dividend and the total wealth (instead of $p_{t}$ ) are similarly ordered. Furthermore, when there is more than one asset, our approach provides pricing bounds for productive assets as well as for derivative assets on any underlying asset and not only on the index. More precisely, it suffices to observe the index process and the terminal payoff of any asset in order to derive pricing bounds on this asset with our method.

In addition to the equity claim described above, there are $m$ purely financial assets (that is to say, assets with total supply always equal to zero). For $i=$ $1, \ldots, m$, the $i^{\text {th }}$ financial asset is tradable at each date and has a price $q_{t}^{i}$ in terms of consumption good.

Formally, we consider a model with finitely many states and dates, where all random processes share a common filtered probability space in which $P$ is the (true) probability and $E_{t}$ [.] denotes the expectation conditional on what is known at $t$. We denote by $\Sigma_{t}$ the set of all date $t$-nodes and, for $\sigma_{t} \in \Sigma_{t}, f\left(\sigma_{t}\right)$ is the set of the immediate successors of the date $t$-node $\sigma_{t}$.

There are $N$ consumers. The $n^{\text {th }}$ consumer has a Von Neumann-Morgenstern utility function $U^{n}($.$) , which associates with any consumption process \left(C_{t}\right)_{0 \leq t \leq T}$, the following utility level at date 0 :

$$
U^{n}(C)=E\left[\sum_{t=0}^{T} u^{n}\left(C_{t}, t\right)\right]
$$

where $u^{n}$ maps $\mathbb{R}^{+*} \times\{0, \ldots, T\}$ in $\mathbb{R}$. We assume the following classical properties on $u^{n}$ :

For all $n, u^{n}$ is continuously differentiable, increasing and strictly concave. Moreover, we impose the following Inada condition

$\forall t, t=0, \ldots, T, \quad u^{n}(x ; t) \underset{x \rightarrow 0^{+}}{\rightarrow}-\infty$.

The sequence of events is: firstly, the firm produces and distributes the dividends among the shareholders; secondly, consumption, new portfolios and new prices take place. The prices come from the equilibrium conditions, and as usual, are considered as given for the agents in their utility maximization program. 
A trading strategy $S$ is a vector

$$
\left\{\left(C_{t}\right)_{t=0, \ldots, T} ;\left(\theta_{t}\right)_{t=1, \ldots, T} ;\left(\alpha_{t}\right)_{t=1, \ldots, T}=\left(\left(\alpha_{t}^{i}\right)_{i=1, \ldots, m}\right)_{t=1, \ldots, T}\right\}
$$

where:

- $\forall t, t=0, \ldots, T, C_{t}$ depends on the information available at date $t$;

- $\forall t, t=1, \ldots, T, \theta_{t}$ and $\alpha_{t}$ depend on the information available at date $t-1$.

We interpret $\theta_{t}$ (resp. $\alpha_{t}^{i}$ ) as the quantity of equity claim (resp. $i^{\text {th }}$ purely financial asset) owned at date $t$ by the agent who follows strategy $S$. Hence, $\theta_{0}$ and $\alpha_{0}$ are the initial quantities of assets owned by the agent. We take, by convention, $\alpha_{T+1}^{i}=\theta_{T+1}=0$.

We denote by $\alpha_{t}^{\prime} . q_{t}$ the inner product between $\alpha_{t}$ and $q_{t}$. The budget constraints at dates $t=0, \ldots, T$ are then:

$$
C_{t}+\theta_{t+1} p_{t}+\alpha_{t+1}^{\prime} \cdot q_{t}=\theta_{t}\left(p_{t}+d_{t}\right)+\alpha_{t}^{\prime} \cdot q_{t}=W_{t}
$$

where $W_{t}$ is interpreted as the wealth before consumption, at date $t$.

For a given agent $n$ with an initial endowment $W_{0}^{n}$, we can define the convex set of the admissible strategies $\mathcal{A}^{n}$ as the set of the strategies $S$ satisfying the budget constraint (3.1) and the consumption constraint $C_{t} \geq 0$ for all $t$ between 0 and $T$.

By definition, a state-price deflator is an adapted stochastic process $\varsigma$ so that, for all $t$ between 0 and $T-1$, we have:

$$
\begin{aligned}
& p_{t}=\frac{1}{\varsigma_{t}} E_{t}\left[\varsigma_{t+1}\left(p_{t+1}+d_{t+1}\right)\right] \\
& q_{t}=\frac{1}{\varsigma_{t}} E_{t}\left[\varsigma_{t+1} q_{t+1}\right]
\end{aligned}
$$

In the following, we will denote by $\Xi$ the set of the state-price deflators. This set is non-empty since we assume that there is an equilibrium. It is well known that, at the equilibrium and from the first-order optimal conditions, the process $\left(\varsigma_{t}^{n}\right)_{t=0, \ldots, T}$ defined, for any agent $n$, by :

$$
\varsigma_{0}^{n}=1
$$

and for all $t$ between 0 and $T-1$ :

$$
\frac{\varsigma_{t+1}^{n}}{\varsigma_{t}^{n}}=\frac{\left(u^{n}\right)^{\prime}\left(C_{t+1}^{n, *}, t+1\right)}{\left(u^{n}\right)^{\prime}\left(C_{t}^{n, *}, t\right)}>0
$$


is a state-price deflator.

If we suppose that the stochastic evolution of the underlying asset price and the prices at the terminal date of the purely financial assets (i.e. their payoffs) $q_{T}$ are known exogenously, our problem consists in how to use those primitives in order to restrict the initial prices of the purely financial assets $q_{0}$ which are compatible with an equilibrium. The classical procedure would be to use the set $\Delta$ of state-price deflators for the stock price process, i.e. the set of the adapted stochastic processes $\varsigma$ so that, for all $t$ between 0 and $T-1$, we have:

$$
p_{t}=\frac{1}{\varsigma_{t}} E_{t}\left[\varsigma_{t+1}\left(p_{t+1}+d_{t+1}\right)\right]
$$

and to remark that, by a no-arbitrage condition, one must have, for all $i$ between 1 and $m$ :

$$
q_{0}^{i} \in\left[\inf _{\varsigma \in \Delta} \frac{1}{\varsigma_{0}} E\left[\varsigma_{T} q_{T}^{i}\right] ; \sup _{\varsigma \in \Delta} \frac{1}{\varsigma_{0}} E\left[\varsigma_{T} q_{T}^{i}\right]\right]
$$

We call this interval the no-arbitrage interval.

For a given node $\sigma_{t} \in \Sigma_{t}$, and for every pair $\left(\sigma^{\prime}, \sigma^{\prime \prime}\right)$ of successors of $\sigma_{t}$, we will say that a given state-price deflator is in reverse order of $d_{t+1}$ on $\left(\sigma^{\prime}, \sigma^{\prime \prime}\right)$ if we have

$$
\left(\varsigma\left(\sigma^{\prime}\right)-\varsigma\left(\sigma^{\prime \prime}\right)\right)\left(d_{t+1}\left(\sigma^{\prime}\right)-d_{t+1}\left(\sigma^{\prime \prime}\right)\right) \leq 0 .
$$

Then, the following lemma holds:

Lemma 1. At the equilibrium, for every node and for every pair $\left(\sigma^{\prime}, \sigma^{\prime \prime}\right)$ of successors of this node, there exists a state-price deflator in reverse order of $d_{t+1}$ on $\left(\sigma^{\prime}, \sigma^{\prime \prime}\right)$.

Proof. See Appendix.

This result implies that for every pair $\left(\sigma^{\prime}, \sigma^{\prime \prime}\right)$, there exists a state-price deflator $\varsigma$ which satisfies the ordering property, and not that there is one state-price deflator $\varsigma$ which satisfies the ordering property for any $\left(\sigma^{\prime}, \sigma^{\prime \prime}\right)$. Of course, if the set of admissible state-price deflators is in fact reduced to one process (complete market at the equilibrium), we get back Perrakis and Ryan's ordering property, as a special case. We recall that we need not observe the price processes which complete the market in order to have this result (see Bizid, Jouini and Koehl (1999)).

Henceforth, we denote by $\Xi_{\left(\sigma^{\prime}, \sigma^{\prime \prime}\right)}$ the set of all the state-price deflators for the stock-price process that are in reverse order of $d_{t+1}$ on $\left(\sigma^{\prime}, \sigma^{\prime \prime}\right)$. 
Theorem 2. Consider a date $t$-node $\sigma_{t}$, with $t$ in $\{0, \ldots, T-1\}$. We suppose that the price values $q_{t+1}^{i}$ of the $i^{t h}$ financial asset (where $i \in\{1, \ldots, m\}$ ) are known for the successors of $\sigma_{t}$. Then, $q_{t}^{i}\left(\sigma_{t}\right)$ must lie in the interval

$$
\left[\max _{\left(\sigma^{\prime}, \sigma^{\prime \prime}\right)} \inf _{\varsigma \in \Xi_{\left(\sigma^{\prime}, \sigma^{\prime \prime}\right)}} \frac{1}{\varsigma_{t}} E_{t}\left[\varsigma_{t+1} q_{t+1}^{i}\right] ; \min _{\left(\sigma^{\prime}, \sigma^{\prime \prime}\right)} \sup _{\varsigma \in \Xi_{\left(\sigma^{\prime}, \sigma^{\prime \prime}\right)}} \frac{1}{\varsigma_{t}} E_{t}\left[\varsigma_{t+1} q_{t+1}^{i}\right]\right]
$$

where the infimum and the supremum are taken over $\Xi_{\left(\sigma^{\prime}, \sigma^{\prime \prime}\right)}$ and the maximum and the minimum are taken over all the pairs of successors of $\sigma_{t}$.

Proof. See Appendix.

Interval (3.3) is not equal to the following interval :

$$
\left[\inf _{\varsigma \in \cap \Xi_{\left(\sigma^{\prime}, \sigma^{\prime \prime}\right)}} \frac{1}{\varsigma_{t}} E_{t}\left[\varsigma_{t+1} q_{t+1}^{i}\right] ; \sup _{\varsigma \in \cap \Xi_{\left(\sigma^{\prime}, \sigma^{\prime \prime}\right)}} \frac{1}{\varsigma_{t}} E_{t}\left[\varsigma_{t+1} q_{t+1}^{i}\right]\right]
$$

Indeed, this last interval (which is Perrakis and Ryan's one) is in fact much more restrictive and does not mean anything in an incomplete market framework. However, we can state the following corollary due to Bizid, Jouini and Koehl (1999):

Corollary 3 (Perrakis-Ryan) Consider a date $t$-node $\sigma_{t}$, for $t \in\{0, \ldots, T-1\}$. We suppose that the price values $q_{t+1}^{i}$ of the $i^{t h}$ financial asset (where $i \in\{1, \ldots, m\})$ are known for the successors of $\sigma_{t}$. If the market is complete after the introduction of the financial assets, then $q_{t}^{i}\left(\sigma_{t}\right)$ must lie in the interval

$$
\left[\inf _{\varsigma \in \cap \Xi_{\left(\sigma^{\prime}, \sigma^{\prime \prime}\right)}} \frac{1}{\varsigma_{t}} E_{t}\left[\varsigma_{t+1} q_{t+1}^{i}\right] ; \sup _{\varsigma \in \cap \Xi_{\left(\sigma^{\prime}, \sigma^{\prime \prime}\right)}} \frac{1}{\varsigma_{t}} E_{t}\left[\varsigma_{t+1} q_{t+1}^{i}\right]\right]
$$

where the infimum and the supremum are taken over all the state-price deflators for the stock-price process that are in reverse order of $d_{t+1}$ on $\left(\sigma^{\prime}, \sigma^{\prime \prime}\right)$ for all pairs of successors of $\sigma_{t}$.

Proof. See Appendix.

In an equilibrium framework (with incompleteness), the state-price deflators satisfy:

$$
\exists(i, j) \in\left\{1, \ldots, f\left(\sigma_{t}\right)\right\}^{2}, i<j, \quad \varsigma_{t+1}(i) \leq \varsigma_{t+1}(j)
$$


It appears then that in the complete market case, it suffices to restrict our attention to state-price densities that are "globally" in reverse order with $d_{t+1}$ :

$$
\begin{aligned}
\varsigma_{t+1}(1) & \leq \varsigma_{t+1}(2) \leq \ldots \leq \varsigma_{t+1}\left(f\left(\sigma_{t}\right)\right) \\
\text { with } d_{t+1}(1) & \geq d_{t+1}(2) \geq \ldots \geq d_{t+1}\left(f\left(\sigma_{t}\right)\right)
\end{aligned}
$$

instead of looking to all the state price densities sets satisfying one of the constraints $\varsigma_{t+1}(i) \leq \varsigma_{t+1}(j)$ for some pair $(i, j)$ such that $d_{t+1}(i) \geq d_{t+1}(j)$.

Let us now go back to the incomplete market case. Since, for each asset $i \in\{1, \ldots, m\}$, the pay-off $q_{T}^{i}$ is assumed to be known, we can apply Theorem 2. We are now in position to compute by backward induction, as for instance in Ritchken (1985), an interval in which $q_{0}^{i}$ must lie ; we will call this interval the equilibrium pricing interval. This result gives bounds on the equilibrium price of any financial asset, only with the knowledge of the dividend process and of the claim price process (interpreted as a market index).

\section{Adding Assets to the Information Set}

In the previous sections, we assumed that the price process of the equity claim was known exogenously and we found equilibrium restrictions on the price processes of the purely financial assets, given their values at the final date $T$. In this section, we suppose as given exogenously not only the price process of the equity claim $p$, but also the price processes of the $\bar{m}$ first purely financial assets $\left(q^{i}\right)_{i=1, \ldots, \bar{m}}$, with $\bar{m}<m$. Using the same approach as above, we want to restrict the possible values of $\left(q^{i}\right)_{i=\bar{m}+1, \ldots, m}$. We are particularly interested in the special case where $\bar{m}=1$ and where the exogenous price process of the first purely financial asset corresponds to a risk-free asset.

Adapting the results of the previous sections, we define the set of state-price deflators for the known price processes, i.e. the adapted stochastic processes $\varsigma$ so that, for all $t$ between 0 and $T-1$, we have:

$$
p_{t}=\frac{1}{\varsigma_{t}} E_{t}\left[\varsigma_{t+1}\left(p_{t+1}+d_{t+1}\right)\right]
$$

and for $i=1, \ldots, \bar{m}$,

$$
q_{t}^{i}=\frac{1}{\varsigma_{t}} E_{t}\left[\varsigma_{t+1} q_{t+1}^{i}\right]
$$


Obviously, this set is contained in the one defined by the no arbitrage condition for the equity claim only. Therefore, for any asset $i$ between $\bar{m}+1$ and $m$, the arbitrage pricing interval is defined as follows:

$$
q_{0}^{i} \in\left[\inf \frac{1}{\varsigma_{0}} E\left[\varsigma_{T} q_{T}^{i}\right] ; \sup \frac{1}{\varsigma_{0}} E\left[\varsigma_{T} q_{T}^{i}\right]\right]
$$

where the infimum and the supremum are taken on the set of state-price deflators for the known price processes. Using Lemma 1 and the fact that every stateprice deflator is, in particular, a state-price deflator for the known price processes, we can apply the methodology of Theorem 2. It is then possible to restrict the interval in which the price of the $m-\bar{m}$ last purely financial assets must lie. As above, we will call this interval the equilibrium pricing interval.

We apply now this result to the special case where $\bar{m}=1$ and $q^{1}$ is a strictly positive predictable process. Then we can define the adapted process $\left(r_{t}\right)_{t=0, \ldots, T-1}$ by

$$
1+r_{t}=\frac{q_{t+1}^{1}}{q_{t}^{1}}
$$

In other words, $q^{1}$ is a risk-free asset, and $r_{t}$ the risk-free rate between dates $t$ and $t+1$. Then, the set $\Delta$ of state-price deflators for the known price processes is equal to

$$
\Delta=\left\{\varsigma ; \varsigma_{t}=\left(1+r_{t}\right) E_{t}\left[\varsigma_{t+1}\right] \text { and } p_{t}=\frac{1}{\varsigma_{t}} E_{t}\left[\varsigma_{t+1}\left(p_{t+1}+d_{t+1}\right)\right]\right\}
$$

The following proposition is the equivalent of Theorem 2 when there exists a risk-free asset with an exogenously given price process. The proof is very close to that of Theorem 2; therefore, it is omitted. The main difference is that we must now work on the risk-neutral probabilities and not on the state-price deflators. For the rest, it is the very same as the previous sections: we prove that we can price the derivatives with a sub-class of the risk-neutral probabilities consistent with the no-arbitrage condition. These risk-neutral probabilities are linked to $\Delta$ as follows: for a fixed $\varsigma \in \Delta$, let us define $\widehat{P}$ by $\prod_{t=0}^{T-1}\left(1+r_{t}\right) \frac{\varsigma_{T}}{\varsigma_{0}}$, its density with respect to $P$ (note that this quantity is strictly positive, which implies that $\widehat{P}$ is equivalent to $P$ ). Furthermore, between a given date- $t$ node $\sigma_{t}$ and one of its successors, the transition probability $\widehat{\pi}$ induced by $\widehat{P}$ must then be equal to the transition probability $\pi$ induced by $P$ multiplied by $\left(1+r_{t}\right) \frac{\varsigma_{t+1}}{\varsigma_{t}}$. 
Since $\varsigma$ defines a state-price deflator for the known price processes, it is easy to see that $\widehat{P}$ is a martingale-measure for the stock price process normalized by the risk-free price process and augmented by the dividends. More precisely we have:

$$
p_{t}\left(\sigma_{t}\right)=\frac{1}{1+r_{t}} \sum \widehat{\pi}(\sigma)\left(p_{t+1}+d_{r+1}\right)(\sigma)
$$

where the sum is taken over all successors of $\sigma_{t}$.

For a given pair $\left(\sigma^{\prime}, \sigma^{\prime \prime}\right)$ of successors of $\sigma_{t}$ so that $d_{r+1}\left(\sigma^{\prime}\right)<d_{r+1}\left(\sigma^{\prime \prime}\right)$ we will say that $\widehat{P}_{\left(\sigma^{\prime}, \sigma^{\prime \prime}\right)}$ is in reverse order of $d_{t+1}$ on $\left(\sigma^{\prime}, \sigma^{\prime \prime}\right)$ if

$$
\frac{\widehat{\pi}\left(\sigma^{\prime \prime}\right)}{\pi\left(\sigma^{\prime \prime}\right)} \leq \frac{\widehat{\pi}\left(\sigma^{\prime}\right)}{\pi\left(\sigma^{\prime}\right)}
$$

The following holds:

Proposition 4. Let us assume that the price processes of the equity claim $p$ and of a risk-free asset (with a total supply always equal to zero) $q^{1}$ are given exogenously, and define $1+r_{t}=\frac{q_{t+1}^{1}}{q_{t}^{1}}$, for $0 \leq t \leq T-1$. Then for all $i$ between 2 and $m$, given $q_{t+1}^{i}$, we get that $q_{t}^{i}\left(\sigma_{t}\right)$ must lie in :

$$
\left[\max _{\left(\sigma^{\prime}, \sigma^{\prime \prime}\right)} \inf _{\widehat{P}_{\left(\sigma^{\prime}, \sigma^{\prime \prime}\right)}} \frac{1}{1+r_{t}} E_{t}^{\widehat{P}_{\left(\sigma^{\prime}, \sigma^{\prime \prime}\right)}}\left[q_{t+1}^{i}\right] ; \min _{\left(\sigma^{\prime}, \sigma^{\prime \prime}\right)} \sup _{\widehat{P}_{\left(\sigma^{\prime}, \sigma^{\prime \prime}\right)}} \frac{1}{1+r_{t}} E_{t}^{\widehat{P}_{\left(\sigma^{\prime}, \sigma^{\prime \prime}\right)}}\left[q_{t+1}^{i}\right]\right]
$$

where the infimum and the supremum are taken on the martingale measures $\widehat{P}_{\left(\sigma^{\prime}, \sigma^{\prime \prime}\right)}$ that are in reverse order of $d_{t+1}$ on $\left(\sigma^{\prime}, \sigma^{\prime \prime}\right)$ and where the maximum and the minimum are taken over all pairs $\left(\sigma^{\prime}, \sigma^{\prime \prime}\right)$ of successors of $\sigma_{t}$.

\section{An Example of Stochastic Volatility Model}

In this section, we investigate numerically an example in a quadrinomial framework. We consider as given the prices of the equity claim $p$ and of a risk-free asset $q^{1}$ and the final pay-offs of the $m-1$ other purely financial assets $q_{T}$. Then, using Proposition 4, we compute the arbitrage pricing and equilibrium pricing intervals for each purely financial asset by backward induction.

We consider a $n$ time-steps lattice. Then, the underlying random process is $\left(p_{k T / n}\right)_{k=0, \ldots, n}$, which distribution is assumed to be known. The discount rate between two successive dates $r T / n$ is assumed to be constant. 
We consider the following reconnecting tree-structure, which models the evolution of the equity claim. If its value at date $t$ is $p_{t}$, then

$$
\left\{p_{t} u_{1}(1+r T / n), p_{t} u_{2}(1+r T / n), p_{t} u_{2}^{-1}(1+r T / n), p_{t} u_{1}^{-1}(1+r T / n)\right\}
$$

are the four possible values of $p_{t+T / n}+d_{t+T / n}$ at date $t+T / n$, with

$$
u_{1}=e^{\sigma_{\max } \sqrt{\frac{T}{n}}} \text { and } u_{2}=e^{\sigma_{\min } \sqrt{\frac{T}{n}}}
$$

The tree structure is stationary, and we assume the ordering principle with respect to the price process instead of the dividend process.

The true transition probabilities are denoted $\left.\left\{\pi_{i}\right\}_{i=1, \ldots, 4} \in\right] 0 ; 1\left[{ }^{4}\right.$.

Let $\left\{\widehat{\pi}_{i}\right\}_{i=1, \ldots, 4} \in[0 ; 1]^{4}$ which satisfy $\sum_{i=1}^{4} \widehat{\pi}_{i}=\sum_{i=1}^{4} \widehat{\pi}_{i} u_{i}=1$. The existence of such $\left\{\widehat{\pi}_{i}\right\}_{i=1, \ldots, 4}$ is guaranteed by the no-arbitrage conditions.

In the rest of the paper, we explain how to use our method for the numerical obtention of the equilibrium pricing interval. We also compare this interval with the arbitrage pricing interval and the interval obtained by Perrakis and Ryan's methodology (denoted Perrakis' pricing interval).

Computations of the equilibrium pricing interval are difficult when the true transition probabilities are not explicitly given. In the following, we consider two concrete examples of true transition probabilities.

\section{A. Equiprobable states of the world}

First assume that $\forall i=1, \ldots, 4, \pi_{i}=\frac{1}{4}$. We have to solve 6 linear optimization problems respectively defined by the conditions $\frac{\widehat{\pi}_{1}}{\pi_{1}} \leq \frac{\widehat{\pi}_{2}}{\pi_{2}}, \frac{\widehat{\pi}_{2}}{\pi_{2}} \leq \frac{\widehat{\pi}_{3}}{\pi_{3}}, \frac{\widehat{\pi}_{3}}{p 3} \leq \frac{\widehat{\pi}_{4}}{\pi_{4}}$, $\frac{\widehat{\pi}_{1}}{\pi_{1}} \leq \frac{\widehat{\pi}_{3}}{\pi_{3}}, \frac{\widehat{\pi}_{1}}{\pi_{1}} \leq \frac{\widehat{\pi}_{4}}{\pi_{4}}$ and $\frac{\widehat{\pi}_{2}}{\pi_{2}} \leq \frac{\widehat{\pi}_{4}}{\pi_{4}}$. Since all the problems are linear, it suffices to determine the extreme points of the polyhedron defined by the constraints and then to maximize on these points.

We obtain the following intervals of prices for a European Call at the money with $K=100, T=1$ year, $r=0 \%$ and for varying values of $\left[\sigma_{\min } ; \sigma_{\max }\right]$. 


\section{TABLE 1}

\begin{tabular}{|c|c|c|c|}
\hline \multicolumn{4}{|c|}{ Call Price Bounds for a varying range of $\left[\sigma_{\min }, \sigma_{\max }\right]$} \\
\hline$\left[\sigma_{\min } ; \sigma_{\max }\right]$ & Perrakis' pricing & Equilibrium pricing & Arbitrage pricing \\
\hline$\overline{[05 \% ; 20 \%]}$ & {$[5.7991 ; 5.8264]$} & {$[4.0180 ; 6.4859]$} & {$[1.9895 ; 7.9457]$} \\
\hline$[06 \% ; 19 \%]$ & {$[5.6058 ; 5.6298]$} & {$[4.2778 ; 6.1860]$} & {$[2.3873 ; 7.5496]$} \\
\hline$[07 \% ; 18 \%]$ & {$[5.4367 ; 5.4574]$} & {$[4.4887 ; 5.9090]$} & {$[2.7851 ; 7.1534]$} \\
\hline$[08 \% ; 17 \%]$ & {$[5.2907 ; 5.3083]$} & {$[4.6575 ; 5.6527]$} & {$[3.1827 ; 6.7569]$} \\
\hline$[09 \% ; 16 \%]$ & {$[5.1714 ; 5.1855]$} & {$[4.7879 ; 5.4245]$} & {$[3.5803 ; 6.3604]$} \\
\hline$[10 \% ; 15 \%]$ & {$[5.0800 ; 5.0906]$} & {$[4.8842 ; 5.2326]$} & {$[3.9778 ; 5.9636]$} \\
\hline$[11 \% ; 14 \%]$ & {$[5.0198 ; 5.0265]$} & {$[4.9458 ; 5.0899]$} & {$[4.3752 ; 5.5667]$} \\
\hline$[12 \% ; 13 \%]$ & {$[4.9857 ; 4.9881]$} & {$[4.9698 ; 5.0086]$} & {$[4.7725 ; 5.1697]$} \\
\hline
\end{tabular}

We consider here a quadrinomial reconnecting tree, which models an equity claim diffusion, which volatility belongs to a range $\left[\sigma_{\min } ; \sigma_{\max }\right]$. We assume that the number of time steps of the model is $n=100$. An important information comes from the true probability distribution. In this Table, we give results coming from our approach and from no-arbitrage conditions for a European call at the money, with a varying range of volatilities. We give also for guidance, results coming from Perrakis' method, even if they are out of context, in an incomplete market. It is clear that the equilibrium bounds are not as precise as Perrakis' ones, but this is due to the fact that we did not suppose arbitrarily the completeness of the market, and then, the possibility to be completely hedged "in Vega" (i.e. against volatility).

As remarked in Bizid, Jouini and Koehl (1999), the very good results for Perrakis' pricing interval come in part from the fact that the true transition probabilities make the price process of the equity claim near to a martingale under the true probability. Indeed, as the states of the world are supposed equiprobable, the ratio $E\left[p_{T}\right] / p_{0}$ is very close to one. In this example, the risk premium for the equity claim is approximately equal to $1 \%$ for one year, which is in fact very low.

\section{B. Non-equiprobable states of the world}

\section{One polar example}

It is interesting to investigate a polar example: $\pi_{1}=\frac{1}{2}, \pi_{2}=\frac{1}{4}, \pi_{3}=\frac{1}{8}$ and $\pi_{4}=\frac{1}{8}$. For instance, with $n=100$ steps, the ratio $E\left[p_{T}\right] / p_{0}$ is equal to 2.0017 . The equity claim risk premium is then about $100 \%$ for one year. Therefore, instead of computing Perrakis' bounds, which suppose the equiprobability of the states of 
the world, we adapted here his results to a non-equiprobable situation, as done in Bizid, Jouini and Koehl (1999).

We calculate the pricing interval for a European Call at the money with $K=$ $100, r=0 \%, T=1$ year, $\left[\sigma_{\min } ; \sigma_{\max }\right]=[10 \% ; 15 \%]$ using a backward induction as in the previous example.

\begin{tabular}{lccc}
\hline \multicolumn{5}{c}{ TABLE 2} \\
Convergence Results for the Call with a high risk premium \\
\hline & $\underline{n=10}$ & $\underline{n=100}$ & $\underline{n=1000}$ \\
Perrakis' & {$[4.7137 ; 5.5578]$} & {$[4.6853 ; 5.5418]$} & {$[4.6890 ; 5.5369]$} \\
Equilibrium & {$[4.6331 ; 5.6630]$} & {$[4.6332 ; 5.6788]$} & {$[4.6418 ; 5.6786]$} \\
Arbitrage & {$[3.8895 ; 5.8312]$} & {$[3.9778 ; 5.9636]$} & {$[3.9868 ; 5.9770]$} \\
\hline
\end{tabular}

We use the same methodology as in the previous example : a recombining quadrinomial tree structure where the volatility of the underlying asset belongs to a range $\left[\sigma_{\min } ; \sigma_{\max }\right]=[10 \% ; 15 \%]$. We give the intervals of prices for a varying number of time-steps $n$ in the lattice. We investigate here a polar example where the risk premium for the equity claim is about $100 \%$ for one year. We give as before, for information, the interval associated with Perrakis' method (equivalent to a complete market situation), which has been adapted to a non-equiprobable context.

This example shows (at least heuristically) that:

- on the contrary to the complete markets case (Perrakis' interval), the precision of the equilibrium pricing interval seems to be less affected by the value of the equity claim risk premium.

- the completeness hypothesis does not permit one to improve the accuracy of the method for high values of the equity claim risk premium.

This example shows the importance of the equity claim risk premium in a derivative asset pricing.

\section{Varying the risk premium}

In the following example, we vary the value of the risk premium as done in Bizid, Jouini and Koehl (1999). We consider varying true probabilities depending linearly on a parameter $\varepsilon \in \mathbb{R}$, as follows: $\pi_{1}=\frac{1}{4}, \pi_{2}=\frac{1}{4}+\varepsilon, \pi_{3}=\frac{1}{4}, \pi_{4}=\frac{1}{4}-\varepsilon$. In this case, we can easily draw the risk premium as a function of the parameter $\varepsilon$ (see Figure 2). 


\section{FIGURE 2}

Risk Premium for the stock vs a measure of the risk premium (parameter $\varepsilon$ )

We consider a quadrinomial stationary tree, representing the binomial volatility model. We assume that the probability, in the true world, for the risky asset to be at a given state, depends linearly on a parameter $\varepsilon$. More specifically, we assume that, at each node, as e increases to $1 / 4$, the transition probability associated with the lowest possible value decreases to 0 and the transition probability of the second possible value increases to $1 / 2$. As this parameter increases, the expected return (or equivalently the risk premium) of the underlying asset increases also (almost linearly). This means that, as e increases, the true probability moves away from the martingale case (i.e. which has no risk premium). We represent here the risk premium when holding the risky asset for one year.

Note that $\varepsilon$ lies by definition in the following closed interval $[-0.0033,0.25]$. Figure 3 represents the pricing bounds for the European Call (with $\sigma_{\min }=10 \%$, $\sigma_{\max }=15 \%$ and a 100 time-steps tree) and varying $\varepsilon$. Perrakis' bounds widen continuously as $\varepsilon$ increases (i.e. as the true probability moves away from the martingale case). For the equilibrium interval, however, this phenomenon is less important.

\section{FIGURE 3}

Bounds of a European ATM call price vs a measure of the risk premium

(parameter $\varepsilon$ )

This Figure illustrates that the bounds of a European Call option price widen as $\varepsilon$ (which defines the distribution of the true probability) increases. We consider more specifically an "At The Money" Call with one year maturity, in a stochastic volatility model, as a function of $\varepsilon$. In fact, only the lower bound of the Call price varies much, because the Call price is an increasing function of the stock price. In this example, the constraint associated with the complete market case is $\varepsilon=0.1483$. The constraint on the probabilities associated with the incomplete market case is for $\varepsilon=0.0485$. Note the exact match for the lower boundaries between an incomplete and a complete market if parameter $\varepsilon$ is greater than 0.1483 .

\section{Conclusion}

In this paper we have shown that the equilibrium conditions give strong restrictions on the admissible martingale-measures and then on the asset prices compat- 
ible with a large class of utility functions : namely, the non-decreasing, concave, Von Neumann-Morgenstern ones. Our results are based upon ordering properties on the state prices as in Perrakis (1986) and Perrakis and Ryan (1984) and involve the true probability as in Jouini, Koehl and Touzi (1999).

We do not assume any completeness assumption on the financial market nor the existence of any completion at the equilibrium (such an existence is equivalent to the equality of the marginal utilities over all the agents). Our bounds are then larger than Perrakis' bounds and this permits us to establish that the set of prices compatible with an equilibrium for a given asset in an incomplete market is larger than the set of all possible prices for that asset compatible with the equilibrium in at least one completion.

In all the paper, we assumed that there is only one productive asset but it is easy to generalize our method to a model with many productive assets and many derivatives on those assets. To do so, it suffices to replace the process $\left(p_{t}\right)$ by the total wealth process or by the index process considered as a proxy of the total wealth.

\section{Appendix}

Proof of Lemma 1. For any date $t$ and any $\sigma_{t} \in \Sigma_{t}$, the total consumption of the economy is $d_{t}\left(\sigma_{t}\right)$ (remember that $\sum_{n=1}^{N} \theta_{t}^{n}=1$ and $\sum_{n=1}^{N} \alpha_{t}^{n}=0$ ).

Then, for a given pair $\left(\sigma^{\prime}, \sigma^{\prime \prime}\right)$ of successors of $\sigma_{t}$ such that $d_{t+1}\left(\sigma^{\prime}\right)>d_{t+1}\left(\sigma^{\prime \prime}\right)$, there exists (at least) one agent $n_{0}$ such that $C_{t+1}^{n_{0}, *}\left(\sigma^{\prime}\right) \geq C_{t+1}^{n_{0}, *}\left(\sigma^{\prime \prime}\right)$. Note that $n_{0}$ depends on $\sigma_{t}$.

Consider the state-price deflator associated with the $n_{0}^{t h}$ agent, as defined in (3.2). As $u^{n_{0}}$ is concave and increasing, we get:

$$
0<\left(u^{n_{0}}\right)^{\prime}\left(C_{t+1}^{n_{0}, *}\left(\sigma^{\prime}\right), t+1\right) \leq\left(u^{n_{0}}\right)^{\prime}\left(C_{t+1}^{n_{0}, *}\left(\sigma^{\prime \prime}\right), t+1\right)
$$

And multiplying both terms of the inequality by a positive value depending only on $\sigma_{t}$, we get

$$
\varsigma_{t+1}^{n_{0}}\left(\sigma^{\prime}\right) \leq \varsigma_{t+1}^{n_{0}}\left(\sigma^{\prime \prime}\right)
$$

Then $\varsigma^{n_{0}}$ is in reverse order of $d_{t+1}$ on $\left(\sigma^{\prime}, \sigma^{\prime \prime}\right)$ and is a state-price deflator by construction.

Proof of Theorem 2. Let fix $i$ in $\{1, \ldots, m\}$ and a node $\sigma_{t} \in \Sigma_{t}$. Applying Lemma 1, for a given pair $\left(\sigma^{\prime}, \sigma^{\prime \prime}\right)$ of successors of $\sigma_{t}$, we obtain the existence of 
a state-price deflator $\varsigma$ in reverse order of $d_{t+1}$ on $\left(\sigma^{\prime}, \sigma^{\prime \prime}\right)$ and we must have

$$
q_{t}^{i}\left(\sigma_{t}\right) \in\left[\inf _{\varsigma \in \Xi_{\left(\sigma^{\prime}, \sigma^{\prime \prime}\right)}} \frac{1}{\varsigma_{t}} E_{t}\left[\varsigma_{t+1} q_{t+1}^{i}\right] ; \sup _{\varsigma \in \Xi_{\left(\sigma^{\prime}, \sigma^{\prime \prime}\right)}} \frac{1}{\varsigma_{t}} E_{t}\left[\varsigma_{t+1} q_{t+1}^{i}\right]\right]
$$

where the infimum and the supremum are taken over all the state-price deflators for the stock-price process that are in reverse order of $d_{t+1}$ on $\left(\sigma^{\prime}, \sigma^{\prime \prime}\right)$. Using the same argument for any pair of successors of $\sigma_{t}$, we obtain that $q_{t}^{i}\left(\sigma_{t}\right)$ must lie in

$$
\underset{\left(\sigma^{\prime}, \sigma^{\prime \prime}\right)}{\cap}\left[\inf _{\varsigma \Xi_{\left(\sigma^{\prime}, \sigma^{\prime \prime}\right)}} \frac{1}{\varsigma_{t}} E_{t}\left[\varsigma_{t+1} q_{t+1}^{i}\right] ; \sup _{\varsigma \in \Xi_{\left(\sigma^{\prime}, \sigma^{\prime \prime}\right)}} \frac{1}{\varsigma_{t}} E_{t}\left[\varsigma_{t+1} q_{t+1}^{i}\right]\right]
$$

where the intersection is taken over all pairs of successors of $\sigma_{t}$. This gives the bounds of equation (3.3) and ends the proof.

Proof of Corollary 3. Let fix $i$ in $\{1, \ldots, m\}$ and a node $\sigma_{t} \in \Sigma_{t}$. Applying Lemma 1, for a given pair $\left(\sigma^{\prime}, \sigma^{\prime \prime}\right)$ of successors of $\sigma_{t}$, we obtain the existence of a state-price deflator $\varsigma$ in reverse order of $d_{t+1}$ on $\left(\sigma^{\prime}, \sigma^{\prime \prime}\right)$. Furthermore, since the market is complete, we know that there exists only one state-price deflator. It must then be in reverse order of $d_{t+1}$ on $\left(\sigma^{\prime}, \sigma^{\prime \prime}\right)$ for all pairs of successors of $\sigma_{t}$ and we must have

$$
q_{t}^{i}\left(\sigma_{t}\right) \in\left[\inf _{\varsigma \in \cap \Xi_{\left(\sigma^{\prime}, \sigma^{\prime \prime}\right)}} \frac{1}{\varsigma_{t}} E_{t}\left[\varsigma_{t+1} q_{t+1}^{i}\right] ; \sup _{\varsigma \in \cap \Xi_{\left(\sigma^{\prime}, \sigma^{\prime \prime}\right)}} \frac{1}{\varsigma_{t}} E_{t}\left[\varsigma_{t+1} q_{t+1}^{i}\right]\right]
$$

where the infimum and the supremum are taken over all the state-price deflators for the stock-price process that are in reverse order of $d_{t+1}$ on all pairs of successors of $\sigma_{t}$.

\section{References}

[1] Aït-Sahalia, Y., Lo, A.W.: Nonparametric estimation of state-price densities implicit in financial asset prices, Journal of Finance 53, 499-547 (1998).

[2] Avellaneda, M., Levy, A., Paras, A.: Pricing and hedging derivative securities in markets with uncertain volatilities, Applied Mathematical Finance 2, 73-88 (1995). 
[3] Bizid, A., Jouini, E., Koehl, P.F.: Pricing of Non-Redundant Derivatives in a Complete Market, Review of Derivative Research 2 (4), 287-314 (1999).

[4] Black, F., Scholes, M.: The pricing of options and corporate liabilities, J. Political Economy 81, 637-659 (1973).

[5] Breeden, D., Gibbons, M., Litzenberger, R.: Empirical tests of the consumption oriented CAPM, Journal of Finance 44, 231-262 (1989).

[6] Breeden, D., Litzenberger, R.H.: Prices of state-contingent claims implicit in option prices, Journal of Business, 51, 621-651 (1978).

[7] Constantinides, G.: Option Pricing Bounds with Transaction Costs, Working Paper (1993).

[8] Detemple, J., Selden, L.: A general equilibrium analysis of option and stock market interactions, International Economic Review 32 279-303 (1991).

[9] Dybvig, P.: Distributional Analysis of Portfolio Choice, Journal of Business 61, 369-393 (1988a).

[10] Dybvig, P.: Inefficient Dynamic Portfolio Strategies or How to Throw Away a Million Dollars in the Stock Market, Review of Financial Studies 1, 67-88 (1988b).

[11] Duffie, D., Zame, W.: The Consumption-Based Capital Asset Pricing Model, Econometrica 57, 1279-1297 (1989).

[12] Epstein, L.G., Zin, S.E.: Substitution, Risk Aversion, and the Temporal Behavior of Consumption and Asset Returns: A Theoretical Framework, Econometrica 57, 937-969 (1989).

[13] Föllmer, H., Schweizer, M.: Hedging of Contingent Claims under Incomplete Information: in Applied Stochastic Analysis, Davis and Elliott, eds. Gordon and Breach, London New York 389-414 (1991).

[14] Harrison, J.M., Kreps, D.: Martingales and Arbitrage in Multiperiod Securities Markets, Journal of Economic Theory 20, 381-408 (1979).

[15] Huang, C.F., Litzenberger, R.H.: Foundations for Financial Economics, North-Holland, Amsterdam (1988). 
[16] Jouini, E., Kallal, H.: Efficient Trading Strategies in the Presence of Market Frictions, Working Paper 9512, CREST, December (1993).

[17] Jouini, E., Napp, C.: Continuous time equilibrium pricing of nonredundant assets, Working Paper, CREST (1999).

[18] Lintner, J.: The Valuation of Risky Assets and the Selection of Risky Investment in Stock Portfolios and Capital Budgets, Review of Economics and Statistics 47, 13-37 (1965).

[19] Mankiw, N.G., Shapiro, M.D.: Risk and return : Consumption beta versus market beta, The Review of Economics and Statistics 68 (3), 452-459 (1986).

[20] Markowitz, H.M.: Portfolio Selection, Cambridge: Basil Blackwell (1991).

[21] Mehra, R., Prescott, E.C.: The equity premium, a puzzle, Journal of Monetary Economics 15, 145-162 (1985).

[22] Merton, R.: Theory of rational option pricing, Bell Journal of Economics and Management Sci. 4, 141-183 (1973).

[23] Perrakis, S.: Option Bounds in Discrete Time: Extensions and the Pricing of the American Put, Journal of Business 59, 119-141 (1986).

[24] Perrakis, S., Ryan, P.J.: Option Pricing Bounds in Discrete Time, Journal of Finance 39, 519-525 (1984).

[25] Ritchken, P.: On option pricing bounds, Journal of Finance 40, 1219-1233 (1985).

[26] Rubinstein, M.: The valuation of uncertain income streams and the pricing of options, Bell Journal of Economics 7, 407-425 (1976).

[27] Sharpe, W.: Capital Asset Prices: A Theory of Market Equilibrium Under Conditions of Risk, Journal of Finance 19, 395-401 (1964).

[28] Soner, H., Shreve, S., Cvitanić, J.: There is no Nontrivial Hedging Portfolio for Option Pricing with Transaction Costs, Annals of Applied Probability 5, 327-355 (1995). 
[29] Zhou, C.: Informational asymmetry and market imperfections: another solution to the equity premium puzzle, Journal of Financial and Quantitative Analysis 34, 445-464 (1999). 